\title{
Das lexikographische Beispiel in der Parömiographie. Formen und Funktionen
}

\author{
Vida Jesenšek, Abteilung für Germanistik, Philosophische \\ Fakultät, Universität Maribor, Maribor, Slowenien (vida.jesensek@siol.net)
}

Zusammenfassung: Der vorliegende Beitrag bespricht das lexikographische Beispiel im Bereich der Parömiographie (Sprichwortlexikographie). Im Fokus stehen Kriterien zur Identifizierung, Festlegung und Selektion jener kontextueller Sprachdaten, die in der Funktion des lexikographischen Beispiels den lexikographischen Prozess in der modernen Parömiographie systematisch und zweckgemäß unterstützen können. Es wird die Idee verfolgt, dass das lexikographische Beispiel in der Zeit der korpusempirischen Lexikographie, die meist auch elektronische lexikographische Produkte hervorbringt, seinen Status und damit seine Funktionalität geändert hat. Von der traditionellen primär dokumentierend-illustrativen Funktion und Unterstützung der individuellen Sprachkompetenz des Lexikographen wird es zum Ausgangs- und Mittelpunkt des lexikographischen Arbeitsprozesses. Es wird zur empirischen Basis für die lexikographische Beschreibung sprachlicher Phänomene und kann erst daraufhin in seiner tradierten illustrativ-dokumentierenden Funktion mikrostrukturell integriert werden. Interdisziplinär und unter Einbeziehung der phraseologie- und parömiologietheoretischen Kenntnisse zur Semantik, Pragmatik und Grammatik der Sprichwörter werden sodann Aussagen zu Qualitätsmerkmalen von Textstellen ausgearbeitet, anhand deren potentielle lexikographische Beispiele im Bereich der Parömiographie identifiziert, systematisch ausgewertet und selektiert werden können. Abschließend werden die Akzeptabilität und Operationalisierbarkeit der festgelegten Qualitätsmerkmale diskutiert sowie einige weiterführende Forschungsfragen angesprochen. Die Überlegungen gründen auf den Erfahrungen bei der Erarbeitung eines mehrsprachigen parömiographischen Produktes, welches als Dokumentation des aktuellen Sprichwortgebrauchs und zugleich als Lern- und Lehrmaterial in fremdsprachlichen Lernzusammenhängen konzipiert und erarbeitet wurde (SprichWort 2010). Der Artikel will somit zu einer seit 1977 geforderten (vgl. Wiegand 1977) und bisher im Bereich der Parömiographie noch nicht realisierten Ausarbeitung einer Theorie des lexikographischen Beispiels beitragen.

Stichwörter: LEXIKOGRAPHISCHES BEISPIEL, BELEG, BELEGBEISPIEL, LEXIKOGRAPHIE, PHRASEOGRAPHIE, PARÖMIOGRAPHIE, SPRICHWORTLEXIKOGRAPHIE, KORPUSLEXIKOGRAPHIE, METHODOLOGIE, METHODIK, FUNKTIONEN, SPRICHWORT, IDENTIFIZIERUNG, SELEKTION

Abstract: The Lexicographic Example in Paremiography. Forms and Func-
tions. This paper discusses the lexicographic example within the scope of paremiography (prov-
erb lexicography).The focus is on criteria for the identification, determination and selection of those
contextual language data that can, in the functioning of the lexicographic example, systematically
and appropriately support the lexicographic process in modern paremiography. The idea is pur- 
sued that during the time of corpus empirical lexicography, which mostly also yielded electronic products, its status and thus its functionality has been altered. From the traditional primarily documentary illustrative function and support of the individual competence of the lexicographer, it became the starting- and central point of the lexicographic working process. It became the empiric base for the description of linguistic phenomena and can only then be microstructurally integrated into its traditional illustrative documentary function. Interdisciplinarily and through inclusion of the phraseological and paremiographical theoretical knowledge of semantics, pragmatics and grammar of the proverbs, assertions are then developed on the quality characteristics of text passages with the help of which potential lexicographic examples within the scope of paremiography can be identified, systematically evaluated and selected. Finally the acceptability and operationability of the determined quality characteristics are discussed as well as some further research questions addressed. The considerations are based on the experiences from the development of a multilinguistic peromiographical product, that was conceptualized and developed as documentation of the actual proverbial use and also as learning and teaching material in foreign language learning contexts (SprichWort 2010). The article will therefore contribute to the development of a theory of the lexicographic example, since 1977 called for (cf. Wiegand 1977) and until now not yet realized within the scope of paremiography.

Keywords: LEXICOGRPAHIC EXAMPLE, CITATION, CITATION EXAMPLE, LEXICOGRAPHY, PHRASEOGRAPHY, PAREMIOGRAPHY, PROVERB LEXICOGRAPHY, CORPUS LEXICOGRAPHY, METHODOLOGY, METHODICS, FUNCTIONS, PROVERB, IDENTIFICATION, SELECTION

\section{Einleitung}

Verfolgt man die Geschichte der praktischen Lexikographie, so sieht man schnell, dass lexikographische Beispiele (LB) in Prozessen der Wörterbucherstellung seit immer eine bedeutende Rolle gespielt haben. Im Allgemeinen spricht man von Belegen, die entweder als Belegbeispiele (vgl. u.a. Haß 1991a) oder als Textbelege (vgl. u.a. Schlaefer 1990) verstanden werden. Aus historischer Sicht steht die Anwendung von Belegen in der Funktion des LB in unterschiedlichen Traditionen, anhand deren sich ihre jeweils dominante Funktion abspiegelt. ${ }^{1}$ Folgerichtig besteht die Funktionalität des LB im Wesentlichen darin, die Existenz eines Lemma-Zeichens zu begründen und seine semantischen, grammatischen, pragmatischen und sonstigen Charakteristika zu illustrieren und dokumentieren. Die Feststellung gilt für allgemeine wie auch für spezielle Wörterbücher und dementsprechend kann auch die historische Parömiographie (Sprichwortlexikographie) in vielen Fällen eine reiche Belegung einzelner Sprichwörter nachweisen (vgl. Mieder 1989: 1034).

Umso überraschender ist die Beobachtung, dass Studien zur Begrifflichkeit und Funktionalität des LB im lexikographietheoretischen Forschungsfeld bis vor kurzem vergleichsweise selten vorkamen (vgl. u.a. Zöfgen 1994). ${ }^{2}$ Eine intensivere Erforschung dieser »lexikographischen Selbstverständlichkeit« ist erst in der letzten Zeit zugegen; das verstärkte Forschungsinteresse kann wohl 
als eine unausweichliche Folge der gegenwärtigen korpusempirischen Lexikographie verstanden werden. Dabei rückt in den Vordergrund die sog. „Urbild“Tradition des LB (Beleg als Ausgangspunkt der Bedeutungsbeschreibung laut $\mathrm{Ha}$ 1991a) und das LB wird zum zentralen Ausgangspunkt der lexikographischen Arbeitsprozesse erklärt (vgl. u.a. Ďurčo 2006, Hein 2012, Jesenšek 2009 und 2010, Steyer 2010 und 2012, Dräger 2011, Klosa, Töpel und Koplenig 2012). Diese zentrale Position gilt umso mehr für die lexikographische Bearbeitung von festen Wortverbindungen unterschiedlicher Ausprägung, darunter auch für Sprichwörter (SW). Werden SW als spezifische, formal und semantisch komplexe Phänomene, als eigenständige Wortschatz- und/oder Texteinheiten angesehen, so sind sie ohne eine systematisch-analytische Beobachtung und Auswertung ihres textuell und situativ eingebetteten Gebrauchs nur ansatzweise (wenn überhaupt) lexikographisch beschreibbar. Die korpusempirische Parömiographie zielt aber nicht nur darauf, SW anhand der empirischen Datenbasis ausführlich beschreiben zu können; sie hat primär in Aussicht, durch die Auswertung empirischer Sprachdaten "verdeckte Strukturen /zu identifizieren/, die sich dem individuellen Wahrnehmungshorizont bis dato verschlossen haben" (Steyer 2012: 287). Anhand einer repräsentativen empirischen Datenbasis kann einerseits bekanntes Wissen über die SW nachgeprüft werden, andererseits - und das wird hier als entscheidender Mehrwert betrachtet - wird dadurch ermöglicht, das gesamte Variations- und Transformationspotenzial von SW zu ermitteln und zudem neue SW zu identifizieren (Steyer 2012: 288f.). ${ }^{3}$ Auswirkungen auf die Auffassung des LB-Status im lexikographischen Arbeitsprozess liegen somit auf der Hand: korpusermittelte Textausschnitte, die SW enthalten, werden zur Grundlage und zum Ausgangspunkt deren lexikographischen Erfassung und fungieren sodann eventuell auch als LB, die die jeweils vorgesehenen Mikrostrukturen systematisch unterstützen vermögen. Somit rücken zwei wesentliche Fragen in den Vordergrund: (1) welche Qualitäten sollen Textausschnitte nachweisen, damit sie in beiden Funktionen in parömiographischen Arbeitsprozessen adäquat auftreten können und (2) welche ist die Methodik, wonach entsprechende lexikographisch relevante Textausschnitte in der Fülle der verfügbaren textuellen Korpusdaten identifiziert, ausgewertet und ausgewählt werden sollen. Nachdem in Kap. 2 die Begrifflichkeit und Funktionalität des LB in Kürze dargelegt werden, wird in Kap. 3 auf beide Fragestellungen näher eingegangen. Die Überlegungen gehen auf die empirischen Sprachdaten aus dem deutschen Teil der mehrsprachigen parömiologischen Datenbank namens SprichWort (2010) zurück und wollen exemplarisch verstanden werden. ${ }^{4}$

\section{Zum Begriff des lexikographischen Beispiels}

In diesem Kapitel wird das LB in seiner Begrifflichkeit kurz besprochen. Während in Abschnitt 2.1 definitorische und funktionale Aspekte im Allgemeinen erörtert werden, stehen in Abschnitt 2.2 Formen und Funktionen des 
LB in der Parömiographie (und Phraseographie) im Vordergrund der Betrachtung.

\subsection{Definition und Funktionen}

Generalisierende und weit gefasste Definitionen des LB findet man in allen relevanten Referenzwerken zur Lexikographie; stellvertretend dazu sei die Definition aus Engelberg und Lemnitzer (2008: 230) genannt:

Das 1. B. /lexikographische Beispiel/, auch Gebrauchsbeispiel oder Verwendungsbeispiel genannt, ist ein authentischer Beleg oder ein auf die Sprachkompetenz des Lexikographen gestütztes Syntagma, welches ein sprachliches Phänomen oder eine Verwendungsweise eines sprachlichen Zeichens illustriert.

Hervorgehoben werden zwei Aspekte des LB: seine demonstrierend-illustrierende Funktionalität und gleichermaßen seine vorhandene oder fehlende Authentizität. Zöfgen (1994) definiert das LB vor dem Hintergrund der Lernlexikographie und betont seine semantikbezogenen Dimensionen; vom LB will er nur dann reden, "wenn es sich um objektsprachliche, eindeutig auf der parole-Ebene angesiedelte Äußerungen handelt, bei denen das monosemierte Lemma-Zeichen in einem semantisch relevanten Kontext gezeigt wird « (Zöfgen 1994: 184). ${ }^{5}$

Weitere Definitionspräzisierungen bringen lexikographietheoretische Arbeiten zu lexikographischen Mikrostrukturen hervor. Nach Wiegand (1989) lassen sich LB an den Elementen der Mikrostruktur mit dem Angabenstatus ${ }^{6}$ erschließen. Bezogen auf die mikrostrukturelle Beschaffenheit traditioneller Printwörterbuchformate werden mehrere Klassen von solchen Angaben festgelegt und definiert. Deutlich ist eine begriffliche Unterscheidung zwischen Beispiel und Beleg: mit den Beispielangaben werden „Kotexte (meistens für das Lemmazeichen und seine Formen) genannt", während Belegangaben zu den wörterbuchbasisbezogenen Angaben gehören (Wiegand 2006: 98). Fungieren Belegangaben als solche, anhand deren LB erschließbar sind, so werden sie Belegbeispielangaben (auch Belegtextangaben) genannt. Der Aspekt der vorhandenen oder fehlenden Authentizität spiegelt sich in der Unterscheidung von Belegbeispielangaben und Kompetenzbeispielangaben ab; hierzu kommen auch Corpusbeispielangaben, die für weitere Ausführungen noch besonders vom Interesse sein werden.

Wie bereits angedeutet, findet man bis vom kurzem relativ wenige theoretisch ausgelegte Arbeiten zum LB. Auf die Forderung Wiegand's aus dem Jahr 1977, man müsste sich mit dem LB theoretisch und systematisch auseinandersetzen, 7 wurde das Augenmerk hauptsächlich auf seine (lerndidaktische) Funktionalität und anschließend auf die Zusicherung seiner Qualität ausgerichtet.

Eine Theorie des LB weist hauptsächlich zwei Komponenten aus, eine deskriptive und eine normative Komponente (vgl. Wiegand 1977). Die deskriptive Theoriekomponente meint die Korrelation des LB zu anderen Elementen einer konkreten Wörterbuchartikel- oder Datenbankeintragsstruktur, wogegen 
mit der normativen Theoriekomponente „bestimmt werden /soll/, was ein gutes lexikographisches Beispiel ist" (Harras 1989: 607); man befragt also die wünschenswerten Eigenschaften von authentischen und/oder konstruierten Sprachdaten („Wünschbarkeiten“, um mit Hermanns (1988) und Harras (1989) zu sprechen), anhand deren sie an der lexikographischen Relevanz gewinnen.

Über die potentielle Funktionalität von LB wurde bisher verschiedentlich diskutiert. So bezieht sich Martin (1989) auf die LB-Funktionen in einsprachigen Wörterbüchern, ${ }^{8}$ während Zöfgen (1994) eine auf die pädagogischen Ziele ausgerichtete Funktionstypologie "des (Satz)beispiels" mit zehn „idealtypischen“ Fällen erarbeitet. ${ }^{9}$ Funktional ausgerichtet sind auch Arbeiten von Nikula (1986) und Cowie (1989). Beide wollen die vorhandene oder fehlende Authentizität der LB vor dem Hintergrund der Wörterbuchfunktionen beurteilen. Während authentische Belege vor allem zur illustrierenden Demonstration des sprachlich Varianten bzw. Besonderen geeignet seien, dienten konstruierte LB eher zur Illustration des Prototypischen. Cowie (1989) will verschiedene LB-Funktionen darüber hinaus in Abhängigkeit von der aktiven bzw. passiven Ausgerichtetheit des jeweiligen Wörterbuchs verstanden wissen.

Somit wären wir bei der benutzerorientierten Beurteilung der LB-Funktionen, die auch in Hermanns (1988) stark zum Ausdruck kommt. Auf die Frage, wozu ein LB dient, wird aus der Benutzerperspektive geantwortet: ein LB sei „ein Modell, nach dem ich mich beim Sprechen und Verstehen richten kann" (Hermanns 1988: 176), ein LB hat die Funktion, dass es "die mögliche Verwendung eines sprachlichen Zeichens zeigt" (Hermanns 1988: 175). Die tradierte demonstrierend-illustrierende Funktion des LB - wonach dieses anderen Angaben, vor allem der Bedeutungsangabe hierarchisch untergeordnet ist - wird jedoch auch aus der umgekehrten Perspektive betrachtet, wenn er schreibt: „die Regel /Bedeutungsangabe/ dient dazu, das Beispiel verständlich zu machen" (Hermanns 1988: 176).

Dadurch zeigt sich eine Reorientierung in der theoretischen Betrachtung des LB. Die gegenwärtig zum Standard gewordene Korpusbasiertheit der Lexikographie (vgl. u.a. Prinsloo 2009, Müller-Spitzer und Engelberg 2011) und der parallele Medienwechsel zur elektronischen und Internetlexikographie haben zur Folge, dass methodisch-methodologische Fragestellungen zur korpusempirischen Suche nach potentiellen LB zentral geworden sind. Im Fokus stehen Aspekte der korpusanalytischen Identifikation, Auswertung und Auswahl von kontextuellen Sprachdaten, die in lexikographischen Prozessen so einsetzbar sind, dass auf ihrer Basis ein Lemmazeichen (hier ein SW) ausführlich und zuverlässig bearbeitet werden kann und dass sie folgerichtig in der demonstrierend-illustrierenden Funktion als LB auftreten können (vgl. u.a. Klosa 2005, Ďurčo 2006, Steyer 2004, 2010 und 2012, Dräger 2011). Vgl. dazu Dräger (2011: 5): »Der Beleg erhält damit einen neuen Stellenwert, indem er nicht mehr nur belegend aufgeführt wird, sondern zum Informationsgewinn beiträgt«, er gehört zum »Kernbereich eines Wörterbuchs «. ${ }^{10}$

Eine vorläufige Schlussfolgerung zur Begrifflichkeit und Funktionalität 
des LB lässt sich nun folgendermaßen formulieren: LB sind Elemente lexikographischer Mikrostrukturen (bezogen auf Wörterbuchartikel traditioneller Wörterbuchformate und/oder auf Strukturen der Datenbankeinträge). Deren Funktionalität besteht traditionellerweise darin, system- und gebrauchsbezogene Eigenschaften eines Lemmazeichens zu präsentieren und $\mathrm{zu}$ illustrieren und dadurch seine in der Regel vielfältige sprachliche Existenz zu begründen und zu dokumentieren. Ermittelt werden die LB anhand der jeweiligen Wörterbuchbasis; neuerdings anhand der quantitativ-qualitativen analytischen Auswertung umfangreicher Textkorpora. ${ }^{11}$ In der gegenwärtigen korpusempirischen Lexikographie gebührt dem LB allerdings eine zentrale Position, da es zum prinzipiellen Ausgangspunkt der lexikographischen Beschreibung eines sprachlichen Ausdrucks erklärt wird. Folgerichtig sind vorrangig Aspekte der korpusbasierten Sprachdatenermittlung zu klären, um eine operationalisierbare Methodik der Identifikation und Auswahl von lexikographisch relevanten Textausschnitten - potentiellen LB sicherzustellen. ${ }^{12}$

\subsection{Das lexikographische Beispiel in der Parömiographie}

Auch für die Parömiographie (und Phraseographie) gilt die Beobachtung, dass systematische Auseinandersetzungen mit dem LB bisher hauptsächlich vor dem methodisch-theoretischen Hintergrund der korpusempirischen Lexikographie entstanden sind. Das heißt jedoch nicht, dass Phraseographen die Relevanz des LB früher nicht erkannt und stellenweise auch nicht explizit betont hätten. Schemann (1989: 1025f.) hat sich dazu sehr kritisch geäußert:

Die Erkenntnis, daß zu einem idiomatischen Wörterbuch Beispiele gehören, hat sich /.../ gerade in den letzten Jahren zunehmend durchgesetzt. Allerdings sind sie oft /.../ das Gegenteil von dem, was ein Beispiel sein soll: eine Situierung des Idioms und seine gesamte Kontextkonstellation, in der seine semantische, seine pragmatische und seine stilistische Funktion als Einheit anschaulich greifbar wird.

In den wenigen (germanistischen) Arbeiten, die sich ausdrücklich mit der lexikographischen Behandlung von SW auseinandersetzen (vgl. Mieder 1984 und 1999, Grzybek 1992, Kispál 2000 und 2007, Kühn 2003, Durčo 2005) wird die Problematik des LB hauptsächlich aus der wörterbuchkritischen Perspektive behandelt (vgl. Mieder 1999, Kispál 2000), ${ }^{13}$ wogegen seine ausdrückliche Thematisierung im Rahmen der Sprichwortlexikographie m.W. noch aussteht. Eine informative Übersicht über die Parömiographie und ausgewählte sprichwortspezifische Beschreibungsaspekte gibt Kispál (2007). Erwähnt wird die Möglichkeit, dass durch Verwendungsbeispiele und Belege SW-Charakteristika dem Wörterbuchbenutzer nahegelegt werden können, festgehalten wird aber auch, dass dies in den Sprichwörterbüchern eher selten praktiziert wird.

Mit Recht sehen Durčo (2006) und Kispál (2007) in der empirischen Erforschung des (kon)textuellen Vorkommens der SW eine erforderliche Basis 
für die Weiterentwicklung der Sprichwortlexikographie. ${ }^{14}$ Die Möglichkeit, von den korpusempirischen Sprachdaten auszugehen und dazu noch das Medium $\mathrm{zu}$ wechseln, bringt für die moderne Parömiographie entscheidende prozessund produktbezogene Konsequenzen mit sich. Einerseits wird durch die Aufhebung der Platzbeschränkung eine komplexe, mehrdimensionale und von einer prinzipiell offenen Anzahl von LB unterstützte lexikographische SWPräsentation ermöglicht; andererseits basiert die lexikographische Bearbeitung von SW auf kontextuellen korpusermittelten Sprachdaten, die nach einer systematischen quantitativ-qualitativen Auswertung als LB in lexikographische Mikrostrukturen integriert werden können. Der ausschlaggebende Aussagewert solcher Sprachdaten besteht darin, realitätsnahe Einblicke in den aktuellen Sprichwortgebrauch zu geben und somit eine adäquate und komplexe lexikographische Bearbeitung zu ermöglichen. ${ }^{15}$ Hein (2012) konnte an konkreten Beispielen einleuchtend nachweisen, welche Wechselverhältnisse sich im lexikographischen Prozess zwischen einem Textbeleg als Basis für die Ausformulierung einer Angabe und einem Textbeleg in der LB-Funktion herstellen, wenn der Lexikograph konsequent mit korpusermittelten Sprachdaten arbeitet. ${ }^{16}$ Obgleich auch mit Problemen konfrontiert, ermöglicht eine konsequente Arbeit mit Korpusdaten die Identifikation von inhaltlichen und formalen Charakteristika eines SW; der sog. "semantische Kern“ eines SW, seine konnotativpragmatischen Bedeutungsanteile samt illokutiv-kommunikativem Potenzial und ebenso strukturell-grammatische Eigenartigkeiten können weitgehend ermittelt werden. Es ist zu erwarten, dass dadurch die problematischsten aber auch die zentralen Aspekte der Phraseographie samt Parömiographie (i.e. semantische und pragmatische Angaben samt Beispielen und Belegen; vgl. dazu Kühn 2003 und Burger 2009) zufriedenstellend gedeckt werden können.

Im Zentrum weiterer Überlegungen steht somit die Fragestellung, welche Eigenschaften kontextuelle Korpusdaten in Form von Redeteilen bzw. Texteilen (im Folgenden werden sie Textstellen benannt) nachweisen müssen, um als LB in der praktischen Parömiographie adäquat und zweckmäßig fungieren zu können. Aus der Sicht der deskriptiven Theoriekomponente des LB lässt sich diese vorerst ziemlich allgemeine Fragestellung folgendermaßen genauer formulieren:

Welche Eigenschaften müssen Textstellen haben, damit sie in der Erarbeitung parömiographischer Produkte so anwendbar sind, dass sie in demonstrativillustrierender Funktion mit jeweils geplanten und verschiedenartigen mikrostrukturellen Elementen (Angaben) konsistent, sinnvoll und zweckgemäß korrelieren und folgerichtig als LB integriert werden können?

Für die korpusempirische Lexikographie sind jedoch in erster Linie solche Qualitätsmerkmale von Textstellen relevant, die es ermöglichen, diese zur Basis und zum Ausgangspunkt der parömiographischen Bearbeitung eines SW zu erklären. Somit lässt sich die Fragestellung weiterhin spezifizieren:

Welche Eigenschaften müssen Textstellen haben, damit sie im parömiogra- 
phischen Prozess so anwendbar sind, dass sie als empirische Ausgangsbasis dienen, anhand deren Parömiographen lexikographische Aussagen zu einzelnen SW erarbeiten können?

Anhand der Sprachdaten aus dem deutschen Teil der SprichWort-Datenbank (vgl. SprichWort 2010) wird nachfolgend versucht, die gefragten Eigenschaften zu systematisieren. Die lexikographische Perspektive wird dabei sinnvoll und zweckmäßig durch die Perspektive der Phraseologie- und Parömiologieforschung ergänzt, da beide Disziplinen bisher bedeutende, allerdings in der praktischen Lexikographie noch immer wenig berücksichtigte Kenntnisse zur Semantik, Pragmatik und Grammatik von SW hervorbringen konnten. ${ }^{17}$

\section{Das lexikographische Beispiel in einem mehrdimensionalen Beschrei- bungsmodell für SW}

Wie in Abschnitt 2.1 ausgeführt, sind mit der deskriptiven Theoriekomponente des LB seine systematischen Zusammenhänge mit einzelnen Angaben innerhalb lexikographischer Mikrostrukturen gemeint. Folgerichtig sollten LB wörterbuchartikelintern mit einzelnen Angaben sinnvoll korrelieren. Des Weiteren wurde gesagt, dass korpusempirisch ermittelte und systematisch ausgewertete Textstellen als Grundlage für die Angabenformulierung zweckmäßig fungieren sollten. Es handelt sich somit um eine reziproke Perspektiven-Relation, die im Folgenden anhand eines parömiographischen mehrdimensionalen Beschreibungsmodells für SW näher betrachtet wird.

Die gegenwärtige lexikographische Bearbeitung von SW ist bekannterweise mangelhaft; es wird auf verschiedene Defizite hingewiesen, dies vorrangig hinsichtlich der Erfassung semantisch-pragmatischer und grammatischer Daten zum SW-Gebrauch. ${ }^{18}$ Dies mag bestimmt auch an den tradierten Wörterbuchformaten liegen, die eine umfassende und mehrdimensionale lexikographische Darstellung wegen Platzbegrenztheit in der Regel nicht oder nur teilweise ermöglichen und funktional gesehen auch nicht immer anstreben. Es hat sich inzwischen allerdings die Einsicht durchgesetzt, dass eine fachgerechte lexikographische Beschreibung von SW in speziellen parömiologischen Produkten nur mehrdimensional und hochgradig strukturiert erfolgen kann (vgl. u.a. Durčo 2005 und 2006, Hrisztova-Gotthardt 2010, Jesenšek 2010 und 2012). Es wurden bisher mehrere komplexe Beschreibungsmodelle vorgeschlagen, darunter das Modell von Ďurčo (1992 und 2005), welches auch in der hier aktuellen SprichWort-Datenbank angewandt wurde. Modellgerecht werden semantische, pragmatische und formalgrammatische Charakteristika eines SW anhand mehrerer Beschreibungsparameter lexikographisch erfasst. Der Zweck der LB, die im Modell vorausgesetzt werden, besteht darin, lexikographische Angaben zu einzelnen Beschreibungsparametern (im Sinne der Regelformulierungen) so zu unterstützen, dass dadurch die LB-eigene demonstrativillustrierende Funktion realisiert werden kann. Werden sie methodisch transparent korpuserempirisch ermittelt, so fungieren sie zugleich als Grundlage für 
die Angabenformulierung.

Das Wesen einer derartigen mehrdimensionalen SW-Beschreibungsstruktur machen drei in sich komplexe Angabenbereiche aus: der Angabenbereich zur Semantik, der Angabenbereich zur Pragmatik und der Angabenbereich zur Grammatik. Vor dem Hintergrund der inzwischen recht umfangreichen Kenntnisse über die Komplexität der SW-Semantik, über die pragmatischen Gebrauchspräferenzen und/oder -restriktionen sowie über die syntagmatischsyntaktischen und formal-strukturellen Charakteristika einzelner SW, lassen sich notwendige Eigenschaften der korpusermittelten Textstellen annehmen, um zum Ausgangspunkt für die Ausformulierung der lexikographischen Angaben zu werden und zugleich als LB dieselben Angaben gezielt unterstützen zu können. Anhand der konkreten Daten aus der SprichWort-Datenbank (und anhand der deutschen SW) werden sie im Folgenden in der Form von exemplarischen Aussagen dargelegt. ${ }^{19}$

\subsection{Der semantische Angabenbereich}

Der Angabenbereich zur Semantik betrifft vorrangig die denotativen Bedeutungsanteile eines SW. Daraus ergibt sich die Aussage (I):

(I) Eine Textstelle ist dann als LB geeignet, wenn es Ausdrücke/Redeteile enthält, die proto- und stereotypische Eigenschaften dessen zeigen, was das SW benennt:

(1) "Wer A sagt, muss auch B sagen", sagt uns beispielsweise, dass etwas Begonnenes auch weitergeführt werden muss - und fällt der zweite Schritt auch noch so schwer. ${ }^{20}$

Die Textstelle (1) in der Funktion eines LB korreliert mit der Bedeutungsangabe (1a) zum Lemma Wer A sagt, muss auch B sagen, anhand deren Informationen zur Bedeutung des SW nachvollziehbar sind:

(1a) Sagt man dafür, dass begonnene Dinge auch zu Ende geführt oder die Konsequenzen eigener Handlungen auch getragen werden sollten.

Die Aussage lässt sich auch eingehender ausformulieren, z.B. dann, wenn einzelne SW-Bedeutungsanteile zu einer besseren Bedeutungserschließung beitragen können, vgl.:

(I a) Eine Textstelle ist dann als LB geeignet, wenn es Ausdrücke/Redeteile enthält, die enzyklopädische, kulturelle oder historische Aspekte des SW-Inhalts zeigen:

(2) Immer wieder zitiert wird der Spruch "Auge um Auge, Zahn um Zahn" im 2. Buch Mose $(21,24)$ - und auch wenn, was Theologen 
betonen, diese Aussage nur als Gebot zur Wiedergutmachung, nicht als Aufforderung zur Rache gemeint sein mag, so ist dieser Vers doch nicht ganz zufällig sehr missverständlich.

Die Textstelle (2) in der Funktion eines LB korreliert mit der Angabe (2a) zum Lemma Auge um Auge, Zahn um Zahn, anhand deren Informationen zum kulturhistorischen Hintergrund des SW nachvollziehbar sind:

(2a) In den Korpusbelegen wird häufig auf die biblische Herkunft des Sprichworts verwiesen.

\subsection{Der pragmatische Angabenbereich}

Der Angabenbereich zur Pragmatik deckt die jeweilige Gebrauchsspezifik eines SW und kann sich in vielerlei Hinsicht zeigen. ${ }^{21}$ Sie betrifft möglicherweise Sprachhandlungen, die anhand eines SW vollzogen werden (können), weiter präferierte und/oder restringierte Verwendung in bestimmten Situationen und Kontexten, kommunikativen Domänen, Textsorten, eventuelle themengebundene Verwendung u.a.m. ${ }^{22}$ Daraus ergibt sich zunächst eine generelle Aussage zur Geeignetheit einer Textstelle als LB zur SW-Pragmatik:

(II) Eine Textstelle ist dann als LB geeignet, wenn es Ausdrücke/Redeteile enthält, die pragmatische Aspekte eines SW zeigen.

Sie lässt sich weiterhin so spezifizieren, dass jeweils einzelne pragmatische SWCharakteristika genannt werden:

(II 1) Eine Textstelle ist dann als LB geeignet, wenn es Ausdrücke/Redeteile enthält, die das sprachhandlungsbezogene Potential eines SW zeigen:

(3) Nun können wir gemeinsam tanzen und können mit Sicherheit behaupten, dass das Tanzen unser größtes Hobby ist. Also: Wenn es am Anfang nicht klappt - nur nicht aufgeben - Übung macht den Meister.

Das LB (3) korreliert mit der pragmatischen Angabe (3a) zum Lemma Übung macht den Meister, anhand deren die durch das SW realisierte Sprachhandlung ERMUTIGEN erschließbar ist:

(3a) In bestimmten Korpusbelegen wird mit dem Sprichwort eine Ermutigung ausgedrückt, geduldig zu sein und nicht zu schnell aufzugeben.

(II 2) Eine Textstelle ist dann als LB geeignet, wenn es Ausdrücke/Redeteile enthält, die das Vorkommen eines SW in einer präferierten kommunikativen Domäne zeigen:

(4) Vorbeugen ist besser als heilen. Ausgewogenes Essen, genügend 
Schlaf, viel trinken, Bewegung im Freien, Freude und Zufriedenheit alle diese Eigenschaften helfen, den Körper gegen Krankheiten zu stärken.

Das LB (4) korreliert mit der Angabe (4a) zum Lemma Vorbeugen ist besser als heilen, anhand deren Informationen zu der typischen Verwendungsdomäne erschließbar sind:

(4a) In den Korpusbelegen kommt das Sprichwort häufig in der Domäne Gesundheit vor.

(II 3) Eine Textstelle ist dann als LB geeignet, wenn es Ausdrücke/Redeteile enthält, die das Vorkommen eines SW in einer präferierten Textsorte zeigen:

(5) Alles was Sie anpacken gelingt. Waage: Wer wagt, der gewinnt. Venus schenkt Ihnen viele zauberhafte Stunden mit Ihrem Herzblatt.

Das LB (5) korreliert mit der Angabe (5a) zum Lemma Wer wagt, gewinnt, anhand deren Informationen zum textsortenpräferierten Gebrauch nachvollziehbar sind:

(5a) In bestimmten Korpusbelegen wird das Sprichwort in Horoskopen verwendet.

(II 4) Eine Textstelle ist dann als LB geeignet, wenn es Ausdrücke/Redeteile enthält, die das präferierte themenbezogene Vorkommen eines SW zeigen:

(6) Wer auch immer für den Spruch "Wer schön sein will, muss leiden" verantwortlich ist, hatte vermutlich nicht die ästhetisch-plastische Chirurgie vor Augen. Dennoch trifft der Satz. Jede Schönheitschirurgie ist ein Eingriff in den Körper und kann mitunter fatale Folgen haben.

Das LB (6) korreliert mit der Angabe (6a) zum Lemma Wer schön sein will, muss leiden, anhand deren Informationen zum themaspezifischen Gebrauch nachvollziehbar sind:

(6a) In den Korpusbelegen wird das Sprichwort häufig in Verbindung mit dem Thema Schönheitschirurgie verwendet.

(II 5) Eine Textstelle ist dann als LB geeignet, wenn es Ausdrücke/Redeteile enthält, die das Vorkommen eines SW in einer präferierten Situation zeigen:

(7) [...] dem Klub droht der Zerfall. Die Spieler haben sich aufgegeben, 
obwohl noch neun Spiele zu absolvieren sind. Bonhof will sich damit nicht abfinden und forderte sie auf, Zeichen zu setzen. [...] Goalie Robert Enke und Stürmer Markus Feldhoff haben schon ihren Abschied verkündet, die Fans dankten es ihnen mit dem Spruchband: "Die Ratten verlassen das sinkende Schiff".

Das LB (7) korreliert mit der Angabe (7a) zum Lemma Die Ratten verlassen das sinkende Schiff, anhand deren die Einschränkung des SW-Gebrauchs auf eine typische Gebrauchssituation erschließbar ist:

(7a) Mit diesem Sprichwort wird immer eine Kritik am Verhalten der Verantwortlichen ausgedrückt.

(II 6) Eine Textstelle ist dann als LB geeignet, wenn es Ausdrücke/Redeteile enthält, die eine dem SW immanente wertende Sprechereinstellung zeigen:

(8) Der Schulwart [...] weiß ein Lied von den Zuständen in seiner Schule zu singen. Die Fassade wurde bereits renoviert. Aber außen hui, innen pfui: Die Duschen mußten vor etwa neun Monaten gesperrt werden, Fliesen fielen von den Wänden, [...] Wasserabflüsse waren verstopft 1000 Schüler können sich nach dem Turnunterricht nicht duschen.

Das LB (8) korreliert mit der Angabe (8a) zum Lemma Außen hui, innen pfui, anhand deren eine negativ wertende Sprechereinstellung erschließbar ist:

(8a) Sagt man leicht abwertend, wenn etwas oder jemand an der Oberfläche gut oder vielversprechend erscheint, bei genauerer Betrachtung aber nicht ist.

\subsection{Der grammatische Angabenbereich}

Der Angabenbereich zur Grammatik betrifft einerseits formal-strukturelle und andererseits syntagmatisch-syntaktische SW-Eigenschaften. Vorrangig interessiert hierbei das SW-eigene Variabilitätspotenzial; die Variabilität betrifft möglicherweise sowohl die formale SW-Struktur als auch seine syntaktisch-syntagmatische textuelle Einbindung. Vom lexikographischen Interesse ist somit ihr (als usuell angesehenes) lexikalisches, morphologisches, syntagmatisches und syntaktisches Transformationspotenzial. Daraus ergibt sich zunächst eine generelle Aussage zur Geeignetheit einer Textstelle als LB zur SW-Grammatik:

\section{(III) Eine Textstelle ist dann als LB geeignet, wenn es Ausdrücke/Redeteile enthält, die grammatische Aspekte eines SW zeigen.}

Sie lässt sich weiterhin wiederum spezifizieren, und zwar so, dass jeweils ein- 
zelne grammatische SW-Charakteristika genannt werden:

(III 1) Eine Textstelle ist dann als LB geeignet, wenn es Ausdrücke/Redeteile enthält, die das lexikalische Variationspotenzial eines SW zeigen (Komponentenvariabilität):

(9) "Stille Wasser gründen tief", urteilt ein Sprichwort. Ohne die Wahrheitstiefe ergründen zu können, sei die Bedeutung erläutert: Wahrt jemand äußerlich den Schein, wirkt sehr ruhig, ist aber im Innern ganz anders, dann wird er oder sie oft als "stilles Wasser" eingestuft. Verbreitet ist das (Vor)urteil für gewöhnlich gegenüber einsilbigen Frauen.

Das LB (9) korreliert mit der Angabe (9a) zum Lemma Stille Wasser sind tief, anhand deren die usuelle Variabilität des Komponentenbestands eines SW erschließbar ist:

(9a) Stille Wasser gründen tief.

(III 2) Eine Textstelle ist dann als LB geeignet, wenn es Ausdrücke/Redeteile enthält, die das flextionsmorphologische Variationspotential eines SW zeigen (Personalformen, Tempus, Modus, Numerus):

(10) Der Frankfurter Gastronom hat seine eigene Apfeltheorie entwickelt: "Die geraden Jahre sind gute, die ungeraden schlechte Erntejahre." Die Ausnahme bestätigt die Regel: "Es kann auch manchmal anders sein."

Das LB (10) korreliert mit der Angabe (10a) zum Lemma Ausnahmen bestätigen die Regel, anhand deren flexionsgrammatisch variable SW-Formen (hier Numerus) erschließbar sind:

(10a) Die Ausnahme bestätigt die Regel.

(11) Die Scherben brachten in diesem Fall kein Glück. Bei einem Pkw, der in der Limburger Straße 29 in Diez abgestellt war, wurde in der Zeit von Dienstag, 8. Mai, $17 \mathrm{Uhr}$ bis Mittwoch, 9. Mai, 8 Uhr der linke Außenspiegel nach unten gedrückt, wodurch der Spiegel mit Halterung zerbrach.

Das LB (11) korreliert mit der Angabe (11a) zum Lemma Scherben bringen Glück, anhand deren flexionsgrammatisch variable Textrealisierung (hier Tempus) erschließbar ist:

(11a) In den Korpusbelegen wird das Sprichwort häufig in der Vergangenheitsform in andere Sätze eingebettet.

(III 3) Eine Textstelle ist dann als LB geeignet, wenn es Ausdrücke/Redeteile 
enthält, die das syntaktisch-syntagmatische Transformationspotential eines SW zeigen (Passiv-, Imperativ-, Nominalisierungstransformation, Verwendung mit Modalverb, Verwendung als Relativsatz u.a.):

(12) Nach 22 Jahren Vorstandsarbeit, davon 16 Jahre im Amt des Vorsitzenden, hat Wolfgang Müller nicht mehr für den Posten des Vorsitzenden der Bibliser Feuerwehr kandidiert. "Es hat sich in den letzten Jahren gezeigt, dass man nicht gleichzeitig auf zwei Hochzeiten tanzen kann" [...] Die Belastung seines Amtes als Kreisbrandinspektor stelle erhöhte Anforderungen an ihn, sodass er sich außerstande sehe, so für den Verein zu arbeiten, "wie ich es eigentlich möchte".

Das LB (12) korreliert mit der Angabe (12a) zum Lemma Man kann nicht auf zwei Hochzeiten tanzen, anhand deren eine als usuell angesehene syntaktisch variable Textrealisierung (hier eingeleiteter Teilsatz) erschließbar ist:

(12a) In den Korpusbelegen wird das Sprichwort häufig als Teilsatz verwendet und mit , dass' eingeleitet.

(13) Die Niederlage in Salzburg? Abgehakt! Aber nicht vergessen. Das Hagebau Team Tirol träumte vor dem heutigen dritten Kampf gegen Salzburg von süßer Rache.

Das LB (13) korreliert mit der Angabe (13a) zum Lemma Rache ist süß, anhand deren eine als usuell angesehen syntaktisch variable Textrealisierung (hier Konversion) erschließbar ist:

(13a) In den Korpusbelegen wird das Sprichwort häufig nicht als Satz, sondern als Satzglied verwendet.

(14) Erst die Arbeit, dann das Vergnügen: So hielten es jüngst die Mitglieder des [...] Ortsvereins Remagen. Sie schlossen an ihre Jahreshauptversammlung [...] einen gemütlichen Nachmittag mit musikalischer Begleitung an.

Das LB (14) korreliert mit der Angabe (14a) zum Lemma Erst die Arbeit, dann das Vergnügen, anhand deren eine durch die SW-Position gegebene themeneinleitende Funktion erschließbar ist:

(14a) In den Korpusbelegen übernimmt das Sprichwort häufig eine thematisch einleitende Funktion und wird entsprechend mit einem Doppelpunkt markiert.

\section{Fazit und Ausblick}

Anhand des LB im Bereich der Parömiographie wurde im Beitrag die Idee verfolgt, dass dieses in der Zeit der korpusempirischen Lexikographie seinen Sta- 
tus geändert hat: Von der primären und tradierten dokumentierend-illustrativen Funktion und somit der Unterstützung der individuellen Sprachkompetenz des Lexikographen wird es zum Ausgangs- und Mittelpunkt der lexikographischen Arbeitsprozesse. Es wird zur empirischen Datenbasis für die lexikographische Erfassung sprachlicher Phänomene (hier der Sprichwörter) erklärt und es kann zugleich in Korrelation mit dem jeweilig aktuellen Mikrostrukturenprogramm seine tradierte dokumentierend-illustrative Funktion systematisch realisieren. Somit rücken in den Vordergrund des Forschungsinteresses methodisch-methodologische Fragen zu Identifikation, qualitativ-quantitativer Auswertung und Selektion von kontextuellen Korpusdaten (hier Textstellen genannt), die in der Funktion eines LB auftreten können. Dadurch schließe ich mich Schnörch und Storjohann (2012) an, wonach eine kritisch-analytische, gegenstandsbezogene und kompetenzgeleitete Auswertung und Überprüfung von korpusempirischen Daten notwendig ist, wenn in der praktischen Lexikographie Qualität und Zuverlässigkeit angestrebt werden.

Die Ausführungen in Kap. 3 gingen von der phraseologisch-parömiologischen Perspektive aus; es wurde versucht, die phraseologietheoretischen Kenntnisse zum (deutschen) SW und das korpusermittelte Wissen davon, wie SW im (gegenwärtigen) textuellen Gebrauch vorkommen, auf eine systematische Art und Weise mit der Begrifflichkeit des LB in Verbindung zu setzen. Obgleich exemplarisch hat es sich deutlich gezeigt, dass im Fall der modernen hochgradig strukturierten digitalen parömiographischen Produkte die einst angestrebte Multifunktionalität von LB (vgl. Hermanns 1988: 177ff.) nicht mehr vorrangig vom Interesse ist. Vielmehr von Bedeutung sind feinkörnige und auf einzelne Dimensionen der Semantik, Pragmatik und Grammatik ausgerichtete Aspekte der LB, wodurch eine ausdifferenzierte und strukturierte parömiographische Beschreibung und jeweils spezifische illustrierende Belegung ermöglicht wird. Es ist weiterhin zu erwarten, dass die empirischen Korpusdaten neue Beobachtungen und Erkenntnisse zum textuellen SW-Gebrauch werden evidenteren und somit weiterführende Untersuchungen anregen können.

Weiterführende Untersuchungen sind aber auch in parömiographietheoretischer Hinsicht notwendig. Die obige Diskussion der deskriptiven Theoriekomponente des LB im Bereich der Parömiographie eröffnet manche Fragen, auf die man im Detail eingehen sollte. Folgende drei seien hier hervorgehoben:

- Es wäre zu prüfen, inwiefern die obigen Aussagen zur prinzipiellen Geeignetheit von Textstellen als LB fungieren zu können in der praktischen Lexikographie akzeptabel und in konkreten lexikographischen Prozessen operationalisierbar sind. Man kann hierbei mit gutem Grund annehmen, dass semantisch-pragmatische Aspekte des LB eher sprachübergreifend sind, während grammatikbezogene Aspekte einzelsprachlich stärker differieren und somit einzelsprachspezifisch festzulegen sind.

— Dringend zu prüfen wäre die normative Theoriekomponente des LB, die 
hier nicht aufgegriffen wurde. Die Fragestellungen zur Verständlichkeit der korpusermittelten empirischen Sprachdaten, zur potenziell notwendigen lexikographischen Intervention, $\mathrm{zu}$ den angestrebten Qualitätsmerkmalen von LB (Lebensnähe, alltägliche bzw. erkennbare Kommunikationskonstellationen, Angemessenheit und Kontrolle der Lexik u.a.) sind jedoch nur mit Bezug auf konkrete lexikographische Produkte und unter Einbeziehung der Wörterbuchbenutzungsforschung $\mathrm{zu}$ beantworten (vgl. herzu neuerdings Potgieter 2012).

- Es ist notwendig, das Thema mit Bezug auf verschiedene Wörterbuchtypen, -funktionen und -formate $\mathrm{zu}$ erforschen, damit LB spezifische Funktionen in Bezug auf das Informationsprogramm des jeweilig $\mathrm{zu}$ erstellenden Wörterbuchs wahrnehmen und minimalistische bis maximalistische Mikrostrukturenprogramme unterstützen können.

\section{Anmerkungen}

1. Haß (1991a) nennt hierzu vier Traditionen: die „Urbild“-Tradition (Beleg als Ausgangspunkt der Bedeutungsbeschreibung), die Nachweis-Tradition (Beleg als Wahrhaftigkeitsbeweis), die Illustratio-Tradition (Beleg als Veranschaulichung einer Regel) und die Vorbild-Tradition (Beleg als Vorbild zur Nachahmung). Vgl. dazu Zöfgen (1994: 155f.) und seine Diskussion zur Wichtigkeit der sog. belegenden Lexikographie in Europa seit dem 18. Jh.

2. Darüber hinaus stellt man fest, dass auch die Wörterbuchkritik dieses Thema eher spärlich angeht und — bezogen auf einzelne Wörterbücher — ziemlich unsystematisch bewertet (vgl. u.a. Jehle 1990).

3. Vgl. Ďurčo (2006: 4): "große Korpora helfen bei der Suche nach Standardformen und -varianten sowie nach sprichwörtlichen Transformationen«.

4. Die Datenbank ist im Rahmen des gleichnamigen EU-geförderten Forschungsprojekts entstanden (2008-2010). Das Projekt wurde unter der Leitung der Autorin dieses Beitrags (Universität Maribor, Slowenien) und unter Mitwirkung der Universität des Hl. Kyrill und Methodius Trnava (Slowakei), der Universität Zlin (Tschechien), der Universität Szeged (Ungarn), der Technischen Universität Graz (Österreich) und des IDS Mannheim (Deutschland) durchgeführt. Es ging primär um die Konzipierung und Erarbeitung einer onlineLernplattform zur Vermittlung von Sprichwörtern. Die SprichWort-Plattform besteht aus drei Komponenten: einer mehrdimensionalen und mehrsprachigen SprichWort-Datenbank, den Didaktischen Inhalten und der SprichWort-Community (www.sprichwort-plattform.org). Mit je unterschiedlicher Gewichtung sind Projektprodukte auf Fremdsprachenlerner, Fremdsprachenvermittler, Entwickler von e-Lernmaterialien, Parömiologen, Parömiographen und Sprichwort-Interessierte gerichtet. Die einbezogenen Sprachen sind Deutsch, Slowenisch, Slowakisch, Tschechisch und Ungarisch, die zueinander jeweils Nachbarsprachen sind. Unter den innovativen Projektdimensionen u.a. das Modell für eine komplexe und mehrdimensionale lexikographische Bearbeitung von SW betont werden. Für eine ausführliche Projektbeschreibung vgl. Jesenšek (2010).

5. Man sieht, dass das Thema vorrangig im Rahmen der pädagogischen Lexikographie (vgl. u.a. Drysdale 1987, Jehle 1990, Zöfgen 1994, Potgieter 2012) und größerer lexikographischer bzw. 
lexikographisch-didaktischer Vorhaben aufgegriffen wird (vgl. u.a. Schlaefer 1990, Haß 1991 und 1991a, Abel 2000, Klosa 2005, Jesenšek 2009, Dräger 2011, Klosa, Töpel und Koplenig 2012).

6. Angaben sind "funktionale lexikographische Textsegmente, die entweder zum Lemmazeichen oder zu artikelinternen Angaben oder zu Angaben in anderen Artikeln oder zu Textteilen in den Außentexten des Wörterbuches in einer Angabebeziehung stehen, und deren genuiner Zweck darin besteht, daß der potentielle Benutzer aus ihnen entweder lexikographische Informationen über den Wörterbuchgegenstand oder solche über diesen und die Wörterbuchform erschließen kann" (Wiegand 1989: 427).

7. Vgl. Wiegand (1977: 102): »Die Lexikographie benötigt eine Theorie des lexikographischen Beispiels. Diese muß zeigen, wie die bedeutungserläuternde /.../ Funktion lexikalischer Paraphrasen systematisch durch Beispieltypen unterstützt werden kann«.

8. Unterschieden werden syntagmatische, paradigmatische, definitorische, rhetorisch-pragmatische, beweisende, metasprachliche, enzyklopädische, ideologische und Vorbildfunktionen des LB (vgl. Martin 1989: 601ff.).

9. Diese reichen von den Pseudo-, enzyklopädischen und definierenden Beispielen (die Letzteren will er eigentlich nicht als LB verstanden wissen) über bedeutungsdeterminierende und bedeutungsdifferenzierende Beispiele bis zu den kollokationsäquivalenten, grammatikillustrierenden und bedeutungsindifferenten Beispielen (vgl. Zöfgen 1994: 186ff.).

10. Diese Betrachtungsperspektive ist allerdings nicht neu. In der Geschichte der Lexikographie spiegelt sie sich in der Zusammenstellung umfangreicher Belegarchive und genauso in redaktioneller Betreuung der lexikographischen Prozesse ab (vgl. dazu die »Urbild«-Funktion bei Haß 1991a), nur scheint es, dass die oben skizzierte funktional und lerndidaktisch ausgerichtete metalexikographische Diskussion vom Ende des 20. Jh. andere Schwerpunkte gesetzt hat. Vgl. z.B. die Beobachtung zur englischen Lexikographie des 18. Jh. in Atkins und Rundell (2008: 543): "Johnson's dictionary thus embodies the principle that languages should be described on the basis of objective evidence of their use - and this, in a sense, is the primary function of examples of usage: as a source of data from which lexicographers construct their entries. Attaching examples to definitions is a separate process«.

11. Zwischen dem sog. Konsultationsparadigma (korpusbasiert) und Analyseparadigma (korpusdriven) (vgl. Steyer 2004) wird hier nicht explizit unterschieden, zumal in der Praxis (und das gilt auch für das hier zugrundeliegende Projekt SprichWort) beide Vorgehen nicht unbedingt voneinander isoliert angewandt sondern im wechselseitigen Verhältnis zueinander stehen können (vgl. Heine 2012: 178).

12. Ein weites LB-Verständnis, wie in Harras (1989: 608) dargelegt und wonach unter LB auch Angaben von Kollokationen, Phrasemen, syntaktischen Strukturformeln des Typs sich vor jmdm., etw., um jmdn. ängstigen aufgefasst werden, wird hier allerdings nicht vertreten. Das hiesige Verständnis von LB deckt sich allgemein gesehen mit dem Harras'schen „Verwendungsbeispiel“ bzw. mit dem „Satzbeispiel“ Zöfgens (1994).

13. Ebenso knapp in der Reflexion des LB sind manche phraseologische Wörterbücher, u.a. das repräsentative phraseologische Wörterbuch des Deutschen Duden Redewendungen (2008). In den Benutzungshinweisen (2008: 23) wird man hauptsächlich über die technischen Aspekte von »Beispielen und Belegen« informiert (Platzierung, Quellenangaben), während ihre Funktionalität sehr allgemein dargelegt wird: sie bestehe in der Veranschaulichung des Gebrauchs des dazugehörigen Lemmas, somit in der Ergänzung der Bedeutungsangaben 
und folgerichtig der helfenden Unterstützung im DaF-Bereich.

14. Nur so ließe sich nämlich die alte Tradition bewältigen, die Heine (2012: 173) kurz und treffend zusammenfasst: »Die Parömiographie des 20. Jahrhunderts basiert überwiegend auf älteren Sammlungen«.

15. Somit wird die Annahme, dass Sprichwörter über die (gegenwärtigen) Textkorpora nicht oder nur schwer zugänglich seien, wie das in Heine (2012: 181) prognostiziert wird, nicht vertreten. Die Erfahrungen im Projekt SprichWort (2010) sprechen dafür, dass korpusermittelte SW-Daten (für alle beteiligten Sprachen, darunter auch für Deutsch) für den lexikographischen Prozess durchaus zugänglich und lexikographisch relevant sind.

16. Die Arbeit Hein's entstand in Anlehnung an das Projekt SprichWort, im dessen Rahmen die lexikographische SW-Bearbeitung ausschließlich anhand der Auswertung von korpusermittelten Sprachdaten verlief.

17. Die Software-Tools zur Identifikation von "guten« Kandidaten zum LB, sog. GDEX (Good Dictionary Examples vgl. u.a. Husák et al. 2008 und Kilgariff et al. 2008), die etwa im Rahmen von Sketch Engine (http://the.sketchengine.co.uk) entwickelt werden, werden in diesem Beitrag ausgeklammert, vor allem deshalb, weil GDEX bisher m.W. nur für Englisch validiert wurde, der vorliegende Beitrag jedoch von der deutschen Sprache ausgeht.

18. Vgl. hierzu Ďurčo (2005: 144f.): »Ein- und zweisprachige Wörterbücher weisen große Defizite hinsichtlich der semantischen, morphosyntaktischen und kommunikativ-pragmatischen Informationen zur Parömiologie auf /.../ Es gilt zu prüfen, /.../ um welche Aspekte die Beschreibung trotz der notwendigen Wörterbuchökonomie in den Lexika erweitert werden sollte, um adäquate Informationen für den aktiven Gebrauch zu liefern /.../ Es fehlen dabei präziser konstruierte Paraphrasen, die zeigen würden, in welchem Umfang bzw. mit welchen Einschränkungen die Paraphrase ihre Gültigkeit hat und welche zusätzlichen differenzierenden semantischen Merkmale im Umfeld um den semantischen Kern noch eine Rolle spielen.«

19. Es sei allerdings betont, dass die vorliegende Auflistung keinen Anspruch auf Vollständigkeit erhebt; es werden lediglich die wesentlichen Aspekte der lexikographischen SWBeschreibung ausgewählt und anhand der deutschen SW exemplifiziert.

20. Alle exemplarischen Textstellen sind korpusermittelt und entstammen dem deutschen Teil der SprichWort-Datenbank (2010). Unter einfacher Nummerierung (1) werden jeweils datenbankeigene LB angegeben, darauf folgt die entsprechende Bezugsangabe aus dem jeweiligen SW-Eintrag (1a). Auf Quellenangaben, die in der Datenbank konsequent angeführt sind, wird hier verzichtet.

21. Zu den pragmatischen Potenzialen von SW gibt es inzwischen recht viel (germanistische) Literatur, vgl. u. a. Lüger 1999, Umurova 2005, Lewandowska 2008, Burger 2010, Hoffmann 2012.

22. Die hier präsentierten Daten betreffen die ausgewählte parömiologische Datenbank (SprichWort). Weitere korpusanalytische Untersuchungen würden eventuelle zusätzliche od. andersartige od. einzelsprachspezifische Besonderheiten des SW-Gebrauchs ans Licht bringen können.

\section{Literatur}

Abel, Andrea. 2000. Das lexikographische Beispiel in der L2-Lexikographie (am Beispiel eines L2Kontext- und Grundwortschatzwörterbuches. Deutsch als Fremdsprache 37(3): 163-169.

Atkins, B.T. Sue und Michael Rundell. 2008. The Oxford Guide to Practical Lexicography. Oxford/ 
New York: Oxford University Press.

Burger, Harald. 2009. Semantische Aspekte der deutschen Phraseographie: die aktuelle Praxis allgemeine und phraseologische Wörterbücher im Vergleich. Mellado Blanco, Carmen. (Hrsg.). 2009. Theorie und Praxis der idiomatischen Wörterbücher: 23-44. Lexicographica. Series Maior 135. Tübingen: Max Niemeyer.

Burger, Harald. 2010. Phraseologie. Eine Einführung am Beispiel des Deutschen. 4. neu bearbeitete Auflage. Berlin: Erich Schmidt Verlag.

Cowie, Anthony P. 1989. The Language of Examples in English Learners' Dictionaries. James, Gregory (Hrsg.). Lexicographers and their Works: 55-65. Exeter Linguistic Studies 14. Exeter: University of Exeter Press.

Dräger, Marcel. 2011. Der phraseologische Wandel und seine lexikographische Erfassung. Konzept des "Online-Lexikons zur diachronen Phraseologie (OLdPhras)“. Dissertation. Freiburg: Albert-Ludwigs-Universität.

Drysdale, Paul D. 1987. The Role of Examples in a Learner's Dictionary. Cowie, Anthony P. (Hrsg.). 1987. The Dictionary and the Language Learner. Papers from the EURALEX Seminar at the University of Leeds, 1-3 April 1985: 213-223. Lexicographica. Series Maior 17. Tübingen: Max Niemeyer.

Duden Redewendungen. 2008. Wörterbuch der deutschen Idiomatik. 3., überarbeitete und aktualisierte Aufl. 2008. Hrsg. von der Dudenredaktion. Mannheim/Leipzig/Wien/Zürich: Dudenverlag.

Ďurčo, Peter. 1992. Ein Matrixmodell für ein- und zweisprachige phraseologische Wörterbücher. Mair, Christian und Manfred Markus (Hrsg.). 1992. Neue Ansätze in der Kontrastiven Linguistik. Proceedings of the Conference Held at the Leopold-Franzens-University of Innsbruck, Austria, 10-12 May 1991. Vol. I: 261-269. Innsbruck: Universität Innsbruck.

Ďurčo, Peter. 2005. Sprichwörter in der Gegenwartssprache. Trnava: Univerzita sv. Cyrila a Metoda v Trnave.

Ďurčo, Peter. 2006. Methoden der Sprichwortanalysen oder Auf dem Weg zum Sprichwörter-Optimum. Buhofer, Häcky Annelies und Harald Burger (Hrsg.). 2006. Phraseology in Motion I. Methoden und Kritik. Akten der Internationalen Tagung zur Phraseologie (Basel, 2004): 3-20. Hohengehren: Schneider Verlag.

Engelberg, Stefan und Lothar Lemnitzer. 2008. Lexikographie und Wörterbuchbenutzung. 3. Aufl. Tübingen: Stauffenburg.

Grzybek, Peter. 1992. Probleme der Sprichwort-Lexikographie (Parömiographie): Definition, Klassifikation, Selektion. Meder, Georg und Andreas Dörner (Hrsg.). 1992. Worte, Wörter, Wörterbücher. Lexikographische Beiträge zum Essener Linguistischen Kolloquium: 195-223. Tübingen: Max Niemeyer.

Harras, Gisela. 1989. Zu einer Theorie des lexikographischen Beispiels. Hausmann, Franz Josef et al. (Hrsg.). 1989: 607-614.

Haß, Ulrike. 1991. Textkorpora und Belege. Metodologie und Methoden. Harras, Gisela, Ulrike Haß und Gerhard Strauß (Hrsg.). 1991. Wortbedeutungen und ihre Darstellung im Wörterbuch: 212-286. Berlin/New York: Walter de Gruyter.

Haß, Ulrike. 1991a. Zu Bedeutung und Funktion von Beleg- und Kompetenzbeispielen im Deutschen Wörterbuch. Kirkness, Alan, Peter Kühn und Herbert Ernst Wiegand (Hrsg.). 1991. Studien zum Deutschen Wörterbuch von Jacob Grimm und Wilhelm Grimm. Bd. II: 535-594. 
Tübingen: Max Niemeyer.

Hausmann, Franz Josef et al. (Hrsg.). 1989. Wörterbücher. Ein internationales Handbuch zur Lexikographie/Dictionaries. An International Encyclopedia of Lexicography/Dictionnaires. Encyclopédie internationale de lexicographie. Handbücher zur Sprach- und Kommunikationswissenschaft 5.1. Berlin/New York: Walter de Gruyter.

Hein, Katrin. 2012. Zugang zu Sprichwortbedeutung und -gebrauch mit Hilfe von Korpora. Steyer, Kathrin (Hrsg.). 2012. Sprichwörter multilingual. Theoretische, empirische und angewandte Aspekte der modernen Parömiologie: 315-340. Tübingen: Narr.

Heine, Antje. 2012. Empirische Methoden der Phraseologieforschung im Wandel der Zeit. Prinz, Michael und Ulrike Richter-Vapaatalo (Hrsg.). 2012. Idiome, Konstruktionen, „verblümte Rede“. Beiträge zur Geschichte der germanistischen Phraseologieforschung: 165-185. Stuttgart: S. Hirzel Verlag.

Hermans, Fritz. 1988. Das lexikographische Beispiel. Ein Beitrag zu seiner Theorie. Harras, Gisela (Hrsg.). 1988. Das Wörterbuch. Artikel und Verweisstrukturen: 161-195. Düsseldorf/Bielefeld: Schwann-Bagel/Cornelsen-Velhagen u. Klasing.

Hoffmann, Sarah. 2012. Argumentative Strukturen in Sprichwörtern. Bern u.a.: Peter Lang.

Hrisztova-Gotthardt, Hrisztalina. 2010. Vom gedruckten Sprichwörterbuch zur interaktiven Sprichwortdatenbank. Überlegungen zum linguistischen und lexikographischen Konzept mehrsprachiger Sprichwortdatenbanken. Bern u.a.: Peter Lang.

Husák, Milos et al. 2008. GDEX: Automatically Finding Good Dictionary Examples in a Corpus. Bernal, Elisenda und Janet DeCesaris (Hrsg.). 2008. Proceedings of the Thirteenth EURALEX International Congress, Barcelona, Spain, 15-19 July 2008: 425-432. Barcelona: Universitat Pompeu Fabra, Institut Universitari de Lingüística Aplicada.

Jehle, Günter. 1990. Das englische und französische Lernerwörterbuch in der Rezension. Theorie und Praxis der Wörterbuchkritik. Lexicographica. Series Maior 30. Tübingen: Max Niemeyer.

Jesenšek, Vida. 2009. Phraseologische Wörterbücher auf dem Weg zu Phraseologiedatenbanken. Mellado Blanco, Carmen (Hrsg.). 2009. Theorie und Praxis der idiomatischen Wörterbücher. Lexicographica. Series Maior 135: 65-81. Tübingen: Max Niemeyer.

Jesenšek, Vida. 2010. Sprichwörter im Netz. Eine Internet-Lernplattform für das Sprachenlernen. Mellado Blanco, Carmen et al. (Hrsg.). 2010. La fraseografía del S. XXI. Nuevas propuestas para el español y el alemán: 125-148. Berlin: Frank \& Timme.

Jesenšek, Vida. 2012. Sprichwörter aus (kontrastiv-)linguistischer, lexikografischer und didaktischer Sicht: zum Projekt SprichWort. Steyer, Kathrin (Hrsg.). 2012. Sprichwörter multilingual. Theoretische, empirische und angewandte Aspekte der modernen Parömiologie: 275-268. Tübingen: Narr.

Kilgarriff, Alan et al. 2008. GDEX: Automatically Finding Good Dictionary Examples in a Corpus. Bernal, Elisenda und Janet DeCesaris (Hrsg.). 2008. Proceedings of the Thirteenth EURALEX International Congress, Barcelona, Spain, 15-19 July 2008: 425-432. Barcelona: Universitat Pompeu Fabra, Institut Universitari de Lingüística Aplicada.

Kispál, Tamás. 2000. Einige deutsche Sprichwort-Wörterbücher aus der Sicht des DaF-Lerners. Bernáth, Árpád und Gunther Dietz (Hrsg.). 2000. Jahrbuch der ungarischen Germanistik 1999: 163-181. Budapest: Gesellschaft ungarischer Germanisten/Bonn: Deutscher Akademischer Austauschdienst.

Kispál, Tamás. 2007. Sprichwörtersammlungen. Burger, Harald et al. (Hrsg.). 2007. Phraseologie. Ein internationales Handbuch der zeitgenössischen Forschung. 1. Halbband: 414-423. Berlin/New York: 
Walter de Gruyter.

Klosa, Annette. 2005. Belege in elexiko. Haß, Ulrike (Hrsg.). 2005. Grundfragen der elektronischen Lexikographie. elexiko - das Online-Informationssystem zum deutschen Wortschatz: 96-104. Berlin/New York: Walter de Gruyter.

Klosa, Annette, Antje Töpel und Alexander Koplenig. 2012. Zur Funktion und Rezeption von Belegen - Ergebnisse einer Benutzungsstudie zum Onlinewörterbuch elexiko. Sprachwissenschaft 37(1): 93-123.

Kühn, Peter. 2003. Phraseme im Lexikographie-Check. Erfassung und Beschreibung von Phrasemen im einsprachigen Lernerwörterbuch. Lexicographica 19: 97-118.

Lewandowska, Anna. 2008. Sprichwort-Gebrauch heute: ein interkulturell-kontrastiver Vergleich von Sprichwörtern anhand polnischer und deutscher Printmedien. Berlin u.a.: Peter Lang.

Lüger, Heinz-Helmut. 1999. Satzwertige Phraseologismen. Eine pragmalinguistische Untersuchung. Wien: Edition Praesens.

Martin, Robert. 1989. L'exemple lexicographique dans le dictionnaire monolingue. Hausmann, Franz Josef et al. (Hrsg.). 1989: 599-607.

Mieder, Wolfgang. 1984. Geschichte und Probleme der neuhochdeutschen Sprichwörterlexikographie. Wiegand, Herbert Ernst (Hrsg.). 1984. Studien zur neuhochdeutschen Lexikographie: 307-358. Hildesheim: Georg Olms.

Mieder, Wolfgang. 1989. Grundzüge einer Geschichte des Sprichwortes und der Redensart. Besch, Werner et al. (Hrsg.). 1989. Sprachgeschichte. Ein Handbuch zur Geschichte der deutschen Sprache und ihrer Erforschung Bd. 3: 2559-2569. Berlin/New York: De Gruyter.

Mieder, Wolfgang. 1999. Sprichwörter/Redensarten — Parömiologie. Heidelberg: Julius Gross Verlag.

Müller-Spitzer, Carolin und Stefan Engelberg. 2011. Elektronische Lexikografie zwischen Grammatik und Lexikon. Engelberg, Stefan, Anke Holler und Kristel Proost (Hrsg.). 2011. Sprachliches Wissen zwischen Lexikon und Grammatik: 559-572. Berlin/New York: De Gruyter.

Nikula, Henrik. 1986. Wörterbuch und Kontext. Ein Beitrag zur Theorie des lexikalischen Beispiels. Weiss, Walter, Herbert Ernst Wiegand und Marga Reis (Hrsg.). 1986. Textlinguistik contra Stilistik? Wortschatz und Wörterbuch. Grammatische und pragmatische Organisation von Rede?: 187-192. Tübingen: Niemeyer.

Potgieter, Liezl. 2012. Example Sentences in Bilingual School Dictionaries. Lexikos 22: 261-271.

Prinsloo, Daniel J. 2009. The Role of Corpora in Future Dictionaries. Nielsen, Sandro und Sven Tarp (Hrsg.). 2009. Lexicography in the 21st Century. In Honour of Henning Bergenholtz: 181-206. Amsterdam/Philadelphia: John Benjamins. .

Schemann, Hans. 1989. Das phraseologische Wörterbuch. Hausmann, Franz Josef et al. (Hrsg.). 1989: 1019-1032.

Schlaefer, Michael. 1990. Praktische Fragen der Beleglexikographie am Beispiel des Deutschen Wöterbuchs von Jacob und Wilhelm Grimm. Schützeichel, Rudolf und Peter Seidensticker (Hrsg.). 1990. Wörter und Namen. Aktuelle Lexikographie. Symposium Schloß Rauischholzhausen, 25.-27. September 1987: 139-154. Marburg: Hitzeroth.

Schnörch, Ulrich und Petra Storjohann. 2012. Ein Korpus als Garant zuverlässiger lexikografischer Informationen? Eine vergleichende Stichprobenuntersuchung. Vatvedt Fjeld, Ruth und Julie Matilde Torjusen (Hrsg.). 2012. Proceedings of the 15th EURALEX International Congress 2012, Oslo, Norway, 7-11 August 2012: 310-322. Oslo: Universitetet i Oslo, Institutt for lingvistiske og nordiske studier. 
SprichWort. 2010. Eine Internetplattform für das Sprachenlernen. www.sprichwort-plattform.org.

Steyer, Kathrin. 2004. Kookurenz, Korpusmethodik, linguistisches Modell, lexikografische Perspektiven. Steyer, Kathrin (Hrsg.). 2004. Wortverbindungen - mehr oder weniger fest. Institut für deutsche Sprache Jahrbuch 2003: 87-116. Berlin/New York: De Gruyter.

Steyer, Kathrin. 2010. Korpusbasierte Phraseographie. Neue empirische Methoden und Beschreibungsformen. Mellado Blanco, Carmen et al. (Hrsg.). 2010. La fraseografía del S. XXI. Nuevas propuestas para el español y el alemán: 249-277. Berlin: Frank \& Timme.

Steyer, Kathrin. 2012. Sprichwortstatus, Frequenz, Musterbildung. Parömiologische Fragen im Lichte korpusmethodischer Empirie. Steyer, Kathrin (Hrsg.). 2012. Sprichwörter multilingual. Theoretische, empirische und angewandte Aspekte der modernen Parömiologie: 287-314. Tübingen: Narr.

Umurova, Gulnas. 2005. Was der Volksmund in einem Sprichwort verpackt ...: moderne Aspekte des Sprichwortgebrauchs anhand von Beispielen aus dem Internet. Frankfurt am Main u.a.: Peter Lang.

Wiegand, Herbert Ernst. 1977. Nachdenken über Wörterbücher: Aktuelle Probleme. Drosdowski, Günther, Helmut Henne und Herbert Ernst Wiegand (Hrsg.). 1977. Nachdenken über Wörterbücher: 51-102. Mannheim/Wien/Zürich: Lexikographisches Institut.

Wiegand, Herbert Ernst. 1989. Der Begriff der Mikrostruktur: Geschichte, Probleme, Perspektiven. Hausmann, Franz Josef et al. (Hrsg.). 1989: 409-462.

Wiegand, Herbert Ernst. 2006. Angaben, funktionale Angabezusätze, Angabetexte, Angabestrukturen, Strukturanzeiger, Kommentare und mehr. Ein Beitrag zur Theorie der Wörterbuchform. Lexikographica 21: 202-379.

Zöfgen, Ekkehard. 1994. Lernerwörterbücher in Theorie und Praxis. Ein Beitrag zur Metalexikographie mit besonderer Berücksichtigung des Französischen. Lexicographica. Series Maior 59. Tübingen: Max Niemeyer. 